\title{
Produtividade e Eficiência de Vacas Nelore em Pastagem de Brachiaria decumbens Stapf nos Cerrados do Brasil Central ${ }^{1}$
}

\section{Antonio Vieira², José Fernando Piva Lobato ${ }^{3}$, Eduardo Simões Correa ${ }^{4}$, Roberto Augusto de Almeida Torres Junior ${ }^{4}$, Ivo Martins Cezar4}

\begin{abstract}
RESUMO - A produtividade de vacas Nelore foi estimada durante quatro anos em pastagem de Brachiaria decumbens Stapf, com correção inicial da acidez e da fertilidade do solo e livre acesso a uma mistura mineral. Foram determinadas as taxas de prenhez (TP), de natalidade (TN) e de desmama (TD), o peso à desmama dos bezerros (PD), a produção de quilos de bezerros por vaca (P) e a eficiência de produção de bezerro por vaca (E) e por área (EA), bem como o peso e a condição corporal (CC) das vacas antes da estação de parição (PAP e CCP), durante o acasalamento (PMA e CCM) e à desmama (PVD e CCD). A TP média foi de 87,5\%, enquanto a TN e a TD foram de 81,7 e $77,2 \%$, respectivamente, considerando-se todas as vacas expostas a touros. O PD médio foi de $170 \pm 21,89 \mathrm{~kg}$, com a média de $202 \pm 16,48$ dias de idade à desmama. A ordem de parto (OP) influenciou o peso dos bezerros à desmama. Bezerros mais pesados foram produzidos pelas vacas entre a OP3 e a OP9. A P e E por vaca exposta a touro foram de 130 e $32,19 \mathrm{~kg}$, respectivamente, e a EA foi de $192 \mathrm{~kg} / \mathrm{ha}$. Nos períodos PAP, PMA e PVD, os pesos vivos foram de $471 \pm 19,67 \mathrm{~kg}, 405 \pm 21,48 \mathrm{~kg}, 410 \pm 9,35 \mathrm{~kg}$ e as condições corporais 4,45 $\pm 0,30,3,32 \pm 0,44,3,54 \pm 0,19$, respectivamente. A correlação entre peso vivo e condição corporal foi de 0,66 . Vacas Nelore, em pastagem de Brachiaria decumbens Stapf com correção inicial da acidez e fertilidade do solo, apresentaram altos índices reprodutivos e produtivos. Vacas em ordens de parto intermediárias foram mais produtivas que as vacas no início e no final da vida reprodutiva.
\end{abstract}

Palavras-chave: condição corporal, ordem de parto, peso à desmama, produção de bezerros por área, produção de bezerros por vaca, taxa de desmama

\section{Nelore Cows Productivity on Brachiaria decumbens Stapf Pasture on the Cerrado Region of Central Brazil}

\begin{abstract}
The productivity of Nelore cows on Brachiaria decumbens Stapf pasture was measured during four years, in a soil initially improved with limestone and fertilizer and with free access to a mineral mixture. Pregnancy rate (PR), calving rate (CR), weaning rate (WR), calves weaning body weight $(\mathrm{BWC})$, productivity $(\mathrm{P}=\mathrm{kg}$ of calf/cow $)$ and efficiency $(\mathrm{E}=\mathrm{P} / 100 \mathrm{~kg}$ of cow $)$ per cow, productivity per area $(\mathrm{AP}=$ calves $\mathrm{kg} / \mathrm{ha})$, as well as cow body weight and body condition before the calving season (CLW and CBC), at the middle of the mating season (MLW and MBC) and at weaning (WLW and WBC) were evaluated. Mean PR, CR and WR of 87.5, 81.7 and 77.2\%, respectively, were observed. Mean LWC of $170 \pm 21.89 \mathrm{~kg}$ and mean weaning age of $202 \pm 16.48$ days were observed. Significant effect of calving order (CO) on LWC was detected. Cows between CO3 and CO9 weaned the heaviest calves. Values of $130 \mathrm{~kg}$ and $32.19 \mathrm{~kg}$ were observed for P and E, respectively, with AP of $192 \mathrm{~kg} / \mathrm{ha}$. The CLW and CBC, MLW and MBC, and WLW and WBC were of 471 \pm 19.67 $\mathrm{kg}$ and $4.45 \pm 0.30 ; 405 \pm 21.48 \mathrm{~kg}$ and $3.32 \pm 0.44 ; 410 \pm 9.35$ and $3.54 \pm 0.19$, respectively. The correlation between body weight and body condition was of 0.66 . Nelore cows maintained on a tropical pastures of Brachiaria decumbens Stapf, in an initial fertilized soil, had high reproduction level and productivity. Cows with medium calving order had higher productivity than primiparous and older cows.
\end{abstract}

Key Words: calving order, calf production/cow, calf production/area, body condition, weaning rate, weaning weight

\section{Introdução}

A bovinocultura de corte nacional tem apresentado avanços significativos na produtividade dos sistemas pecuários, tanto na fase de recria como de terminação de bovinos de corte (Euclides Filho, 2000).
No entanto, a fase inicial do processo de produção e de criação de raças zebuínas tem sido pouco estudada (Lima, 1974; Barbosa et al., 1978; Aroeira \& Rosa, 1982; Silva et al., 1987). Indicadores que fundamentam a eficiência do processo produtivo na fase de cria, como as taxas de prenhez (TP), de natalidade (TN) e

\footnotetext{
1 Trabalho de pesquisa financiado pela Embrapa/Gado de Corte. Campo Grande, MS.

2 In memorian. Parte da Dissertação de Mestrado do primeiro autor - Departamento de Zootecnia, UFRGS. Pesquisador II da Embrapa/ Gado de Corte, C.P. - 154, Br 262 Km 4, Campo Grande - MS, CEP - CEP: 79002-970, Fone: (67)368-2000. E.mail: avieira@cnpgc.embrapa.br 3 Prof. Adjunto IV, Dep. de Zootecnia-Bolsista IA CNPq, Porto Alegre-RS, C.P. 15.100, CEP: 90.001-970. E. mail: jose.fernando.Iobato@ufrgs.br ${ }^{4}$ Pesquisador da Embrapa/Gado de Corte, C.P. - 154, Br 262 Km 4, Campo Grande - MS, CEP: 79002-970, Fone: (67)368-2000. E.mail: eduardo@cnpgc.embrapa.br; ivocezar@cnpgc.embrapa.br; rtorres@cnpgc.embrapa.br
} 
de desmama (TD), o peso à desmama (PD), a produtividade (P) em quilos de bezerros desmamados/vaca exposta à reprodução e a eficiência (EA) por área destinada à cria, apresentam baixos índices e continuam pouco estudados nos sistemas de produção (Zimmer \& Euclides Filho, 1997; Lobato, 2001).

A produtividade destes sistemas de cria pode ser aumentada pelo uso de processos tecnológicos. Entretanto, na fase de cria, muito pouco do conhecimento gerado é usado e, cada vez mais, em decorrência da expansão da agricultura de grãos em áreas mais agricultáveis, as piores pastagens existentes são destinadas às vacas de cria (Zimmer et al., 1998; Lobato, 2001). A oferta e o valor nutritivo das forrageiras geralmente estão aquém das exigências dos animais, com reflexos diretos na produção final (Euclides Filho, 2000). Assim, as condições nutricional e corporal das vacas ao longo do ano se mantêm abaixo das condições de mantença, resultando em baixo desempenho na fase de cria dos rebanhos de corte (Wiltbank, 1991; Lobato, 2001). Este cenário impossibilita a melhoria do processo reprodutivo, como a desmama de um bezerro/vaca/ano e a elevação da média de peso à desmama. Estudos realizados registraram peso médio à desmama em torno de $150 \mathrm{~kg}$ (Cardellino \& Castro, 1987; Silva et al., 1987; Eler et al., 1989) e baixa taxa de prenhez (Aroeira \& Rosa, 1982).

No entanto, trabalhos com a raça Nelore em regime de pastagens no Centro-Oeste brasileiro, promovendo melhorias no manejo animal, como a recria em pastagens cultivadas, adequação da lotação e estação de monta na reprodução, têm comprovado aumentos significativos nas taxas de prenhez e de natalidade em vacas adultas e em novilhas com primeiro serviço aos dois anos de idade (Pimentel \& Zimmer, 1983; S.Thiago et al., 2000; Corrêa et al., 2001), sem melhorias no peso à desmama.

Estudos indicam ser a redução do ciclo pecuário inviável sem a produção eficiente de bezerros. Sistemas avançados de produção de pecuária de corte dispõem de sistemas de cria com altas taxas de desmama e com maior quantidade de quilos de bezerros desmamados/vaca e por área (Zimmer \& Euclides Filho, 1997; Beretta et al., 2002).

Neste trabalho, avaliou-se a produção e produtividade na fase de cria de um sistema simulado de produção de gado de corte com bovinos da raça Nelore mantidos em pasto com suplementação mineral na região dos cerrados do Centro-Oeste brasileiro.

\section{Material e Métodos}

Empregando-se o modelo de simulação desenvolvido por Cezar (1982), foi estudada a produção de um sistema de cria, procurando-se aumentar o número e o peso de bezerros à desmama. Os principais fatores de produção e produtividade considerados por Cezar (1982) e neste trabalho foram taxa de desmama de $70 \%$, peso à desmama dos machos de $180 \mathrm{~kg}$, idade ao primeiro parto de 36 meses, mortalidade de bezerros/ as de $4 \%$ e das outras categorias de $1 \%$.

O trabalho foi conduzido na Fazenda Modelo, do Centro Nacional de Gado de Corte da EMBRAPA, em Terenos, MS. O solo é do tipo Latossolo Roxo Distrófico (EMBRAPA, 1979), bem drenado, ácido, deficiente em fósforo e microelementos, como o zinco, cobre e enxofre, com presença significativa de alumínio. A vegetação original é do tipo Cerrado e o clima é do tipo Tropical semi-úmido, subtipo AW (Trewartha, 1954), com ocorrência anual de um período seco nos meses mais frios de ano (maio a setembro) e de um período chuvoso nos meses mais quentes (outubro a abril). A precipitação média anual é de $1500 \mathrm{~mm}$, ocorrendo $75 \%$ no período das águas. A temperatura média anual é de $22,9^{\circ} \mathrm{C}$ e a umidade do ar de $72 \%$. Na Tabela 1 , são apresentados os dados meteorológicos do período experimental e as médias obtidas em 29 anos de observações (1973-2001).

A área experimental era constituída de 80 ha de Brachiaria decumbens Stapf. O solo teve correção inicial da acidez com $2.500 \mathrm{~kg} /$ ha de calcário dolomítico (PRNT 80\%) e $500 \mathrm{~kg} / \mathrm{ha}$ da fórmula 5-20-20.

A área foi subdividida em quatro pastos de 20 ha cada, utilizados em pastejo rotacionado, com 14 dias de ocupação e 42 dias de descanso. A média anual de lotação foi de 1,25 vacas/ha. Os animais receberam suplementação mineral composta de $57 \%$ de fosfato bicálcico, $35 \%$ de cloreto de sódio com os micronutrientes sulfato de zinco $0,96 \%$, sulfato de cobre $0,51 \%$, iodato de potássio $0,01 \%$, sulfato de cobalto $0,01 \%$ e selênito de sódio $0,006 \%$.

O rebanho foi estabilizado em 100 vacas de três a 13 anos, média de idade de 5,5 anos e 4,3 crias. Anualmente, 19 novilhas foram incorporadas e acasaladas aos dois anos de idade, para a reposição das vacas de descarte. Após o diagnóstico de gestação, novilhas e vacas não -gestantes mesmo aquelas prenhes excedentes, foram eliminadas. A estação de monta foi de 
Tabela 1 - Dados meteorológicos anuais durante a fase experimental (1997/ 2000) e médias de 29 anos de observação $(1973 / 2001)$

Table 1 - Annual meteorological data during the experimental period (1997/2000) and the mean of 29 years of observations (1973/2001)

\begin{tabular}{|c|c|c|c|c|c|}
\hline \multirow[t]{2}{*}{$\begin{array}{l}\text { Ano } \\
\text { Year }\end{array}$} & \multirow[t]{2}{*}{$\begin{array}{l}\text { Precipitação } \\
\text { Rainfall }\end{array}$} & \multicolumn{3}{|c|}{$\begin{array}{c}\text { Temperaturas } \\
\text { Temperatures }\end{array}$} & \multirow[t]{2}{*}{$\begin{array}{c}\text { Umid. relativa } \\
\text { Moisture }\end{array}$} \\
\hline & & $\begin{array}{l}\text { Mínima } \\
\text { Minimum }\end{array}$ & $\begin{array}{c}\text { Máxima } \\
\text { Maximum }\end{array}$ & $\begin{array}{l}\text { Média } \\
\text { Mean }\end{array}$ & \\
\hline & $\mathrm{mm}$ & \multicolumn{3}{|c|}{${ }^{\circ} \mathrm{C}$} & $\%$ \\
\hline $1973 / 2001$ & 1532,2 & 18,6 & 29,4 & 22,9 & 72 \\
\hline 1997 & 1568,7 & 19,4 & 30,0 & 23,6 & 71 \\
\hline 1998 & 1443,5 & 19,2 & 29,6 & 23,3 & 75 \\
\hline 1999 & 1087,8 & 18,8 & 30,7 & 23,5 & 65 \\
\hline 2000 & 1505,1 & 18,7 & 29,7 & 23,1 & 71 \\
\hline
\end{tabular}

$1^{\circ}$ de novembro a 31 de janeiro do ano seguinte. A desmama dos bezerros ocorreu aos seis/sete meses de idade.

Os touros utilizados eram puros de origem (relação touro/vaca 1:30), aptos a reprodução por exames andrológicos prévios às estações de monta.

No controle sanitário, foram aplicados os procedimentos a seguir: assepsia do umbigo após o nascimento, vacinações contra a febre aftosa, brucelose, carbúnculo sintomático e gangrena gasosa, botulismo, aplicações de vermífugo, controle estratégico da mosca-do-chifre, de berne e carrapato.

Como medidas de eficiência reprodutiva, foram avaliadas durante quatro anos (1997/2000) a TP, a TN e a TD obtidas em relação ao número de fêmeas acasaladas no ano anterior. Na avaliação da taxa de mortalidade, foram consideradas as taxas de mortalidade do nascimento até a desmama e da desmama até a idade adulta.

As determinações de pesos vivos e a condição corporal das vacas, conforme a escala de Lowman et al. (1976), considerando-se ' 1 ' o animal em extremo estado de magreza e ' 5 ' o animal gordo, foram: a) peso (PAP) e condição corporal das vacas (CCP) antes da estação de parição (julho/agosto); b) peso (PMA) e condição corporal das vacas (CCA) durante a estação de acasalamento (dezembro); c) peso (PVD) e condição corporal das vacas (CCD) na desmama dos bezerros (março/abril). $\mathrm{O}$ peso à desmama (PD) dos bezerros foi ajustado para 205 dias de idade (PAJ 205). Foi considerado o peso médio ao nascimento (PN) para os machos de $29 \mathrm{~kg}$ e para fêmeas de $27 \mathrm{~kg}$, conforme Abreu (1949) e Albuquerque et al. (1993).
Os pesos vivos foram obtidos sem jejum prévio, sempre à primeira hora da manhã, na tentativa de se obter resultados próximos das condições de manejo em nível de fazenda.

Para produtividade do rebanho $(\mathrm{P})$ e eficiência das vacas em reprodução (E), foram considerados quilos de bezerros desmamados por vaca e quilos de bezerros desmamados para cada $100 \mathrm{~kg}$ de peso vivo de vacas expostas à reprodução no ano anterior. Para produtividade por área (EA), foi considerada a produção total em quilos de bezerros obtidos no ano em relação à área ocupada pelo rebanho em reprodução.

$\mathrm{Na}$ análise estatística, foram utilizados modelos lineares e logísticos, que se adequaram melhor ao tipo de dados desbalanceados trabalhados, constantes no programa SAS/STAT (1990).

O modelo de análise adotado para avaliação do desempenho reprodutivo das vacas foi:

$\mathrm{Y}_{\mathrm{ijklm}}=\mathrm{m}+\mathrm{A}_{\mathrm{i}}+\mathrm{OP}_{\mathrm{j}}+\mathrm{DP}_{\mathrm{k}}+\mathrm{S}_{1}+\beta * \mathrm{ID}_{\mathrm{ijklm}}+\mathrm{E}_{\mathrm{ijklm}}$, em que $\mathrm{Y}_{\mathrm{ijklm}}=$ característica avaliada na vaca $\mathrm{m}$, com cria do sexo $1(1=1$ e 2$)$, com diagnóstico de prenhez $\mathrm{k}(\mathrm{k}=1$ e 2$)$, com ordem de parto $\mathrm{j}(\mathrm{j}=1,2$, $3, \ldots, 14)$, parida no ano $\mathrm{i}(\mathrm{i}=1,2,3,4,5) ; \mathrm{m}=$ constante associada a todas as observações; $\mathrm{A}_{\mathrm{i}}=$ efeito do ano $\mathrm{i} ; \mathrm{OP}_{\mathrm{j}}=$ efeito da ordem de parto $\mathrm{j} ; \mathrm{DP}_{\mathrm{k}}=$ efeito do dignóstico de prenhez $\mathrm{k} ; \mathrm{S}_{1}=$ efeito do $1_{-}$ésimo sexo da cria; $\beta=$ coeficiente de regressão variável considerada em função da idade da cria à desmama; $\mathrm{ID}_{\mathrm{ijklm}}=$ idade à desmama da cria do sexo 1 , da vaca $\mathrm{m}$, com diagnóstico de prenhez $\mathrm{k}$ e ordem de parto $\mathrm{j}$, parida no ano $\mathrm{i} ; \mathrm{E}_{\mathrm{ijklm}}=$ erro aleatório associado a todas as observações. 


\section{Resultados e Discussão}

Durante o período de 1996/1997 a 1999/2000, foram avaliados os desempenhos reprodutivo e produtivo de 468 fêmeas (Tabela 2).

As vacas em reprodução apresentaram, em média, $87,5 \%$ de TP, $81,7 \%$ de TN e $77,2 \%$ de TD em relação àquelas expostas a touros. Considerando-se somente as vacas mantidas no rebanho após descarte das não-gestantes ou excedentes, as TN e TD foram de 93,0 e $87,0 \%$, respectivamente, superiores àquelas trabalhadas na simulação. Houve $6 \%$ de mortalidade de bezerros, maior que os $4 \%$ simulados. A média das TP obtidas foi superior às taxas encontradas por Santiago et al. (1983), Saueressig \& Rocha (1985) e Corrêa et al. (2000), em rebanhos de cria no Brasil Central, as quais variaram de $60 \%$ a $85 \%$. A TN de $93 \%$ também foi superior à de $92 \%$ encontrada por Leme et al. (1989), em pastagem de Colonião adubada com fósforo, potássio e nitrogênio, e à de $80 \pm 5,7 \%$ de S.Thiago et al. (2000), em pastagem cultivada de Brachiária humidícola não adubada, mas com uso de fósforo suplementar na mistura mineral. As lotações utilizadas nos trabalhos supracitados, com exce- ção de Leme et al. (1989), que utilizaram 1,6 vacas/ha, foram inferiores à de 1,25 vacas/ha do presente trabalho.

Considerando-se a repetição de prenhez das vacas que desmamaram os bezerros a TP foi de $87 \%$ (Tabela 3). As primíparas tiveram TP média de 69\%, enquanto aquelas com duas ou mais crias, de $91 \%$. As diferenças anuais não foram significativas, tanto para as primíparas $\left(\chi^{2}=6,35 ; \mathrm{P}>0,05\right)$ como para vacas adultas $\left(\chi^{2}=4,83 ; \mathrm{P}>0,05\right)$, em virtude do pequeno número de vacas não-gestantes/ano (apenas 11 de 85 vacas, Tabela 3$)$.

Pacola et al. (1983) obtiveram, em vacas primíparas mantidas em pasto ou suplementadas com farelo de algodão (0,513 kg/cabeça/dia; P>0,05), TP de 38,7 e $42,5 \%$, respectivamente. No terceiro parto, a TP foi de 80,0 e $85,0 \%(\mathrm{P}>0,05)$ para as vacas mantidas em pasto e em pasto + suplemento, respectivamente. Neste trabalho, as menores TP ocorreram no ano de $1999 / 00$, de $53 \%$ para as primíparas e de $85 \%$ para as vacas com duas ou mais crias. Em 1999, ocorreu um longo período seco (Tabela 1), comprometendo o crescimento e a qualidade das pastagens e, conseqüentemente, o desempenho reprodutivo do sistema

Tabela 2 - Desempenho reprodutivo das vacas e taxas de mortalidade nos anos avaliados Table 2 - Reproductive performance of cows and mortality rates during the years evaluated

\begin{tabular}{|c|c|c|c|c|c|}
\hline & $1996 / 97$ & $1997 / 98$ & $1998 / 99$ & $1999 / 00$ & Média (Mean) \\
\hline Vacas expostas $\left(\mathrm{N}^{\mathrm{o}}\right)$ & 119 & 120 & 116 & 113 & 117 \\
\hline \multicolumn{6}{|l|}{$N^{o}$ of mating cows } \\
\hline $\mathrm{TP}(P R), \%$ & 88,2 & 90,8 & 91,4 & 79,7 & 87,5 \\
\hline $\mathrm{TN}(C R), \%$ & 84,0 & 87,5 & 87,1 & 68,1 & 81,7 \\
\hline $\mathrm{TD}(W R), \%$ & 82,4 & 80,8 & 82,8 & 62,8 & 77,2 \\
\hline Mortalidade de bezerros (\%) & 2,0 & 8,0 & 5,0 & 8,0 & 6,0 \\
\hline Calves mortality (\%) & & & & & \\
\hline $\begin{array}{l}\text { Mortalidade de adultos (\%) } \\
\text { Adults mortality (\%) }\end{array}$ & 0,7 & 0,7 & - & - & 0,4 \\
\hline
\end{tabular}

$\mathrm{TP}=$ Taxa de prenhez $(P R=$ Pregnancy rate $) ; \mathrm{TN}=$ Taxa de natalidade $(C R=$ Calving rate $) ; \mathrm{TD}=$ Taxa de desmama $(W R=$ Weaning rate $)$.

Tabela 3 - Taxa de prenhez (\%) de vacas primíparas e adultas com desmame de bezerros durante o período experimental

Table 3 - Pregnancy rate (\%) of primiparous and adult cows with weaned calves during the experimental period

\begin{tabular}{|c|c|c|c|c|c|}
\hline $\begin{array}{l}\text { Categoria/ano } \\
\text { Class/year }\end{array}$ & $1996 / 97$ & $1997 / 98$ & $1998 / 99$ & $1999 / 00$ & $\begin{array}{l}\text { Média } \\
\text { Mean }\end{array}$ \\
\hline Primíparas (Primiparous) & $53(8 / 15)^{\mathrm{K}}$ & $76(13 / 17)$ & $88(14 / 16)$ & $53(8 / 15)$ & $69(11 / 16)$ \\
\hline $\operatorname{Vacas}($ Cows $)$ & $93(50 / 54)$ & $95(71 / 75)$ & $93(67 / 72)$ & $85(64 / 75)$ & $91(63 / 69)$ \\
\hline Média (Mean) & $84(58 / 69)$ & $91(84 / 92)$ & $92(81 / 88)$ & $80(72 / 90)$ & $87(74 / 85)$ \\
\hline
\end{tabular}

K- (Vacas prenhas/total); (Pregnant cows/total) 
em teste. McManus et al. (2002) encontraram maiores intervalos de partos e, portanto, menor TP, nos anos em que a disponibilidade de forragem comprometeu o nível nutricional.

A média da TM de bezerros foi de $6 \%$ (Tabela 2) e foi referente aos bezerros já submetidos a tratamentos preventivos do umbigo e identificados. As causas foram diversas, como diarréias e ataque de predadores nas primeiras horas após o nascimento. Leme et al. (1989) encontraram TM de 4\% durante a fase de aleitamento, enquanto Corrêa et al. (2000) observaram $6 \%$ de TM. Madruga et al. (1984) citam como principal causa da mortalidade de bezerros nos primeiros meses de vida a ocorrência de diarréias. A mortalidade de animais adultos foi baixa, inferior a $1 \%$.

As médias dos PV e CC das vacas nos anos avaliados encontram-se na Tabela 4. A média dos quatro anos de peso vivo foi de $429 \mathrm{~kg}$ e a de condição corporal 3,7 .

Os maiores PV e CC $(\mathrm{P}>0,05)$ ocorreram antes do início da parição no mês de agosto (PAP), com 471 $\pm 19,67 \mathrm{~kg}$ e CCP de 4,45 $\pm 0,30$. Em dezembro, no meio do período de acasalamento, os pesos (PMA) e as condições corporais (CCM) foram de $405 \pm 21,48$ $\mathrm{kg}$ e 3,32 $\pm 0,44$, respectivamente. Na desmama, em março/abril, os PVD e CCD foram de $410 \pm 9,35 \mathrm{~kg}$ e 3,54 $\pm 0,19$, respectivamente. Houve perda média de $66 \mathrm{~kg}$ entre PAP e PMA e redução de 1,13 pontos de condição corporal entre CCP e CCM. A partir do PMA até PVD, houve um incremento de 5,0 kg de PV e de 0,22 na CCD (Tabela 4). Na fase de aleitamento das crias, a recuperação da $\mathrm{CC}$ tende a ser lenta, reduzindo o aumento do $\mathrm{PV}$ e a melhoria da $\mathrm{CC}$ no período pós-parto (Wiltbank et al., 1962). Moraes \& Lobato (1993) observaram, em vacas com desmame aos $147 \pm 18,7$ dias e 209 $\pm 22,2$ dias, CC de 3,14 e 2,88 $(\mathrm{P}<0,05)$, no período pré-parto, de 2,77 e 2,86 , no parto, e de 2,80 e 2,77, no acasalamento $(\mathrm{P}>0,05)$, não havendo melhoria na $\mathrm{CC}$ das vacas nos períodos pósparto e de acasalamento.

Embora não tenham sido estatisticamente significativos $(\mathrm{P}>0,05)$, os menores $\mathrm{PV}$ e CC observados ocorreram na PMA de 1999/2000, com médias de 375 $\pm 36,23 \mathrm{~kg}$ e de $2,77 \pm 0,77$, respectivamente, e refletiram nas taxas de prenhez (Tabela 3) no ano de 1999, quando ocorreu baixa disponibilidade e qualidade de forragem, em razão de a precipitação anual ter sido inferior $(108,8 \mathrm{~mm})$ em relação à média de 29 anos (Tabela 1). A matéria seca total foi de $1.357 \mathrm{~kg} / \mathrm{ha}$, com $976 \mathrm{~kg} /$ ha no mês de dezembro.

Uma alternativa para se enfrentar déficits forrageiros é o uso de suplementos. Ruas et al. (2000) observaram aumento de PV e CC de vacas da raça Nelore em pastagem, ao melhorarem a alimentação suplementando-as com um ou dois quilos de ração protéica. Vacas recebendo diariamente $2,0 \mathrm{~kg}$ de ração protéica apresentaram PV e CC de $493 \mathrm{~kg}$ e 4,6, respectivamente. Vacas não-suplementadas tiveram $452 \mathrm{~kg}$ e 4,14 $(\mathrm{P}<0,05)$. Uma segunda alternativa é a redução da carga animal e/ou o desmame precoce dos bezerros (Simeone \& Lobato, 1996; Fagundes et al., 2003; Pötter \& Lobato, 2004), especialmente em vacas primíparas.

Foram desmamados, durante os quatro anos, 348 bezerros, 171 machos e 177 fêmeas. A média de PV foi de $170 \pm 21,89 \mathrm{~kg}$, com média de $202 \pm 16,48$ dias

Tabela 4 - Peso vivo (PV) e condição corporal média (CC) das vacas antes das parições (PAP, CCP), durante as estações de acasalamento (PMA, CCA) e à desmama (PVD, CCD)

Table 4 - Body weight $(B W)$ and body condition $(B C)$ of cows before calving (CLW, $C B C)$, at the middle of the breeding season (MLW, $M B C)$ and at weaning (WLW, WBC)

\begin{tabular}{llcccc}
\hline Ano & & PAP/CCP & PMA/CCM & PVD/CCD & Média \\
Year & & $C L W / C B C$ & $M L W / M B C$ & Mean \\
\hline $1996 / 97$ & $\mathrm{PV}(B W)$ & $463 \pm 45,27$ & $403 \pm 42,33$ & $406 \pm 45,88$ & 424 \\
& $\mathrm{CC}(B C)$ & $4,44 \pm 0,63$ & $3,27 \pm 0,64$ & $3,39 \pm 0,69$ & 3,70 \\
$1997 / 98$ & $\mathrm{PV}(B W)$ & $482 \pm 40,19$ & $420 \pm 41,24$ & $420 \pm 37,15$ & 441 \\
& $\mathrm{CC}(B C)$ & $4,75 \pm 0,43$ & $3,43 \pm 0,65$ & $3,69 \pm 0,60$ & 3,96 \\
$1998 / 99$ & $\mathrm{PV}(B W)$ & $491 \pm 37,33$ & $421 \pm 40,50$ & $415 \pm 40,98$ & 442 \\
& $\mathrm{CC}(B C)$ & $4,56 \pm 0,44$ & $3,83 \pm 0,67$ & $3,73 \pm 0,72$ & 4,04 \\
$1999 / 00$ & $\mathrm{PV}(B W)$ & $447 \pm 43,22$ & $375 \pm 36,23$ & $399 \pm 39,93$ & 407 \\
& $\mathrm{CC}(B C)$ & $4,05 \pm 0,63$ & $2,77 \pm 0,77$ & $3,36 \pm 0,76$ & 4,39 \\
Média & $\mathrm{PV}(B W)$ & $471 \pm 19,67$ & $405 \pm 21,48$ & $410 \pm 9,35$ & 3,77 \\
Mean & $\mathrm{CC}(B C)$ & $4,45 \pm 0,30$ & $3,32 \pm 0,44$ & $3,54 \pm 0,19$ & \\
\hline
\end{tabular}


de idade à desmama. O ganho médio diário (GMD) do nascimento à desmama foi de $0,703 \pm 0,10 \mathrm{~kg}$ e a média do peso ajustado aos 205 dias de idade (PAJ 205), de $172 \pm 20,47 \mathrm{~kg}$ (Tabela 5).

Os PD observados neste trabalho foram superiores aos registrados por Eler et al. (1989), em dados referentes ao desenvolvimento ponderal das provas zootécnicas das raças zebuínas. Esses autores, ao avaliarem 37 rebanhos da raça Nelore no estado de São Paulo, criados exclusivamente em pastagens, obtiveram PAJ 205 dias de $150 \mathrm{~kg}$. Silva et al. (1987), também avaliando animais da raça Nelore, criados em regime de pastagem, encontraram PAJ 205 de $149 \mathrm{~kg}$. Dode et al. (1989) determinaram peso à desmama aos sete meses de idade de $157 \mathrm{~kg}$ criando bezerros Nelore em pastagem de Brachiaria brizantha. Euclides Filho et al. (1998), ao avaliarem vacas Nelore puras e cruzadas, em pastagem de Brachiaria decumbens com suplementação mineral, verificaram PD para bezerros Nelore de $158 \mathrm{~kg}$ e para bezerros mestiços, filhos de vacas $1 / 2$ Fleckvieh $1 / 2$ Nelore, $172 \mathrm{~kg}$ $(\mathrm{P}<0,05)$. Os GMD foram de 0,620 e 0,670 kg, respectivamente $(\mathrm{P}<0,05)$, com média de idade à desmama (ID) de seis meses. Neste trabalho, os resultados foram semelhantes aos obtidos por Euclides Filho et al. (1998) com bezerros mestiços. O melhor desempenho dos bezerros avaliados neste estudo pode ser atribuído à pastagem, em solo previamente corrigido para acidez e fertilidade quando da implantação da pastagem, mantendo-se disponibilidade média de matéria seca total de $2.259 \mathrm{~kg} / \mathrm{ha}$ por ano durante o período experimental (Tabela 6). A disponibilidade média de $1.365 \mathrm{~kg}$ de matéria seca no período 1999 foi suficiente para manter o desenvolvimento e o peso à desmama dos bezerros.

Tabela 5 - Idade (ID), peso à desmama (PD), ganho médio diário (GMD 205) do nascimento à desmama e peso ajustado aos 205 dias (PAJ 205), em quilogramas dos bezerros

Table 5 - Age (WA), weaning weight ( $L W C)$, average daily gain (ADG 205) from birth to weaning and the adjusted weight at 205 days old (ALW 205) in kilograms of calves

\begin{tabular}{lcccc}
\hline Ano & ID & PD & GMD205 & PAJ205 \\
Year & $W A$ & $L W C$ & $A D G 205$ & $A L W 205$ \\
\hline 1997 & $204 \pm 25,59$ & $162 \pm 22,08$ & $0,663 \pm 0,10$ & $164 \pm 21,00$ \\
1998 & $193 \pm 12,73$ & $167 \pm 23,51$ & $0,720 \pm 0,12$ & $175 \pm 24,13$ \\
1999 & $205 \pm 12,92$ & $176 \pm 18,32$ & $0,719 \pm 0,08$ & $176 \pm 16,13$ \\
2000 & $205 \pm 14,68$ & $173 \pm 21,51$ & $0,710 \pm 0,10$ & $173 \pm 20,62$ \\
Média & $202 \pm 16,48$ & $170 \pm 21,89$ & $0,703 \pm 0,10$ & $172 \pm 20,47$ \\
Mean & & & & \\
\hline
\end{tabular}

Reajustando e analisando os dados pelo modelo linear (SAS/STAT,1990) para o peso à desmama (PD), houve efeito de ano, de sexo (SX) e de idade à desmama (ID). Os efeitos de ano não serão discutidos em maiores detalhes, em virtude das condições ambientais distintas em diferentes anos, sem controle experimental destes efeitos. Pelo ajuste do modelo estatístico, o menor peso à desmama ocorreu no ano de 1997, com 161,55 $\pm 3,02 \mathrm{~kg}$, e o maior no ano de 1999 , com $172,78 \pm 2,73 \mathrm{~kg}(\mathrm{P}<0,01)$. A média do peso ajustado pelo modelo foi de $169,75 \pm 17,69 \mathrm{~kg}$. Os machos foram $14,66 \pm 2,01 \mathrm{~kg}$ mais pesados que as fêmeas $(\mathrm{P}<0,01)$. De acordo com Silva et al. (1987), que avaliaram mais de cinco mil bezerros da raça Nelore, os machos à desmama foram $12,5 \mathrm{~kg}$ mais pesados que as fêmeas $(\mathrm{P}<0,01)$. Ferreira \& Sampaio (1981) avaliando um rebanho da raça Nelore durante sete anos, observaram nos machos peso de 9,2 kg superior ao das fêmeas. O PAJ 205, incluindo machos e fêmeas, foi de $162 \mathrm{~kg}$. Houve acréscimo de peso de $0,506 \pm 0,06 \mathrm{~kg}$ para cada dia a mais na idade à desmama dos bezerros $(\mathrm{P}<0,01)$. A análise logística comprovou efeito $(\mathrm{P}<0,01)$ da ordem de parto no peso à desmama dos bezerros (Tabela 7).

Considerando o peso à desmama dos bezerros em relação à ordem de parto, as vacas primíparas (OP1) e aquelas com ordem de parto no final da vida reprodutiva produziram bezerros mais leves que as OP3 a OP9 (Tabela 7). Resultados semelhantes foram determinados por Cardellino \& Castro (1987) e por outros pesquisados por eles citados. Embora não-significativo $(\mathrm{P}>0,05)$, vacas com repetição de prenhez desmamaram bezerros mais leves $(167,50 \mathrm{~kg})$ que as não-gestantes anteriormente $(170,81 \mathrm{~kg})$.

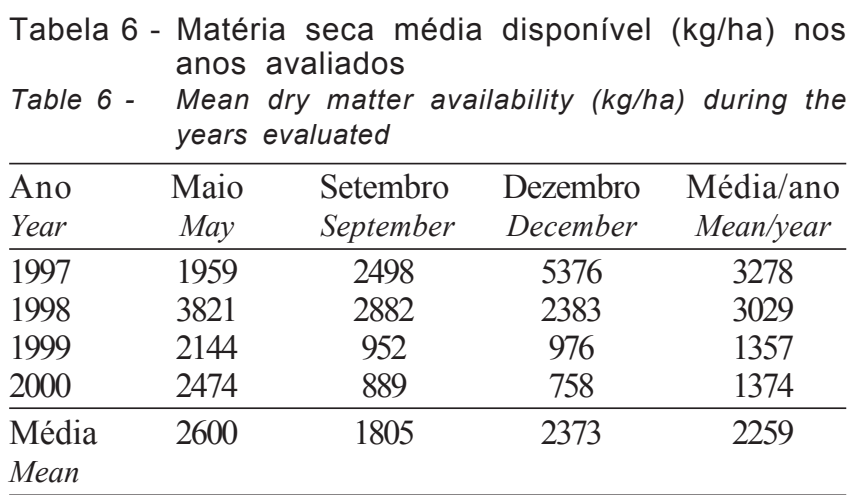


\begin{tabular}{c} 
Tabela 7 - Ordem de parto (OP) e peso à desmama (PD) \\
dos bezerros \\
Table 7 - $\begin{array}{l}\text { Calving order (CO) and calves weaning weight } \\
\text { (LWC) }\end{array}$ \\
\hline
\end{tabular}

Fonte de variação

Source of variation

Média dos quadrados mínimos (PD) \pm desvio-padrão

Least square mean

(LWC) \pm standard deviation

$\mathrm{OP} 1 / \mathrm{CO} 1$

$\mathrm{OP} 2 / \mathrm{CO} 2$

$159,01 \pm 2,30$

$\mathrm{OP} 3 / \mathrm{CO} 3$

$171,24 \pm 3,59$

$\mathrm{OP} 4 / \mathrm{CO} 4$

$178,10 \pm 3,37$

$178,07 \pm 3,44$

OP $5 / C O 5$

OP6/CO6

$176,03 \pm 3,20$

$174,45 \pm 3,69$

OP $7 / C O 7$

$174,34 \pm 3,95$

OP $8 / C O 8$

$163,33 \pm 4,04$

$\mathrm{OP} 9 / \mathrm{CO} 9$

$174,64 \pm 5,17$

OP10/CO10

$169,78 \pm 4,32$

OP $11 / \mathrm{CO} 11$

$162,73 \pm 4,93$

OP $12 / C O 12$

$170,28 \pm 5,43$

OP $13 / \mathrm{CO} 13$

$158,68 \pm 8,04$

OP $14 / C O 14$

$157,50 \pm 17,89$

$R^{2}=0,3966 ; C V=10,42 \%$

Tabela 8 - Produtividade $(P)$ e eficiência $(E)$ por vaca e por hectare (EA) na produção de bezerros, em quilogramas

Table 8 - Productivity (P) and efficiency (E) per cow and per area $(A P)$ in kilograms

\begin{tabular}{lccc}
\hline Ano/Year & $\mathrm{P} / P$ & $\mathrm{E} / E$ & $\mathrm{EA} / A P$ \\
\hline $1997 / 98$ & 129 & 33,18 & 192 \\
$1998 / 99$ & 129 & 31,30 & 193 \\
$1999 / 00$ & 133 & 32,10 & 192 \\
\hline Média (Mean) & 130 & 32,19 & 192 \\
\hline
\end{tabular}

$\mathrm{P}=$ Quilos de bezerro/vaca exposta (Kilograms of calf//mating cow); $\mathrm{E}=\mathrm{P} / 100 \mathrm{~kg}$ de vaca $(E=P / 100 \mathrm{~kg}$ cow $) ; \mathrm{EA}=\mathrm{kg}$ de bezerro $/ \mathrm{ha}$ $(A P=k g$ of calves/ha).

A produtividade e a eficiência do rebanho, considerando-se as vacas expostas ao acasalamento, em quilos de bezerros produzidos por vaca e a eficiência por hectare (EA), avaliadas em um período de três anos, encontram-se na Tabela 8.

Foram registradas média de $\mathrm{P}$ de $130 \mathrm{~kg}$ e de $\mathrm{E}$ de $32,19 \mathrm{~kg}$. Euclides Filho et al. (1995), ao considerarem a relação entre os pesos das vacas e os das crias à desmama, obtiveram E de $41 \mathrm{~kg}$ de bezerro com vacas cruzas $1 / 2$ Fleckvieh $1 / 2$ Nelore e $38 \mathrm{~kg}$ com vacas $1 / 2$ Chianina $1 / 2$ Nelore e $1 / 2$ Charolês $1 / 2$ Nelore $(P<0,05)$.
Euclides Filho (2000) estimou como média nacional para vacas zebuínas E de 25,19 kg, P de $88,16 \mathrm{~kg}$ para peso vivo médio das vacas de $350 \mathrm{~kg}$. Os resultados neste trabalho para $\mathrm{P}$ e E foram superiores em 32,18 e $21,75 \%$ aos obtidos por Euclides Filho et al. (1995) e Euclides Filho (2000). Para a estimativa de E, Euclides Filho et al. (1995) compararam somente as vacas paridas e que desmamaram os bezerros.

Considerando a área destinada à cria, a média da produtividade anual por hectare foi de $192 \mathrm{~kg}$ de bezerro. Euclides Filho et al. (1995), trabalhando com lotação de 1,0 vaca/ha, registraram produções de 200 e $195 \mathrm{~kg}$ para vacas $1 / 2$ Fleckvieh $1 / 2$ Nelore e $1 / 2$ Charolês $1 / 2$ Nelore $(\mathrm{P}>0,05)$ e de $189 \mathrm{~kg}$ para as vacas $1 / 2$ Chianina $1 / 2$ Nelore. As vacas $1 / 2$ Fleckvieh $1 / 2$ Nelore tiveram produção de bezerros superiores as $1 / 2$ Chianina $1 / 2$ Nelore $(\mathrm{P}<0,05)$. Cezar $(1995)$, em condições de pastagem degradada no Brasil Central, verificou produções de $35 \mathrm{~kg}$ de bezerro desmamado/vaca/ha/ano.

No Rio Grande do Sul, Barcellos et al. (1996) obtiveram uma P média de bezerros de 148,3, 122,9 e 82,7 , ao avaliarem vacas primíparas $1 / 2$ Nelore $1 / 2$ Hereford, $1 / 4$ Nelore $3 / 4$ Hereford e Hereford, respectivamente. A E foi de 35,0,31,4 e 21,8 kg, respectivamente. Neste experimento, os resultados para $\mathrm{P}$ e E foram próximos aos observados para vacas primíparas cruzadas e superior aos de primíparas Hereford. Vacas primíparas têm peso vivo inferior aos de multíparas, como as deste trabalho, podendo por isso apresentar uma maior E, embora as crias de vacas primíparas também apresentem tendência para menor peso à desmama. Beretta et al (2001), trabalhando com taxa de natalidade de 82,5 e $92,3 \%$, encontraram produções de bezerros por hectare de 83,9 e 77,9 kg. As lotações consideradas foram de $370 \mathrm{~kg}$ de peso vivo/ha nos meses de primavera, verão e outono e de $170 \mathrm{~kg} / \mathrm{ha}$ no inverno. O peso à desmama considerado foi de $150 \mathrm{~kg}$ aos 180 dias de idade. Mesmo nas condições de altas taxas de natalidade, os resultados obtidos pelos autores foram inferiores aos observados no presente trabalho. A taxa de lotação média na fase de cria, de $1,25 \mathrm{vaca} / \mathrm{ha}$, a taxa de desmama ( $87 \% \mathrm{em}$ relação às vacas presentes no rebanho após descarte) e o peso à desmama (PAJ 205), de $172 \pm 20,47 \mathrm{~kg}$, contribuíram para a maior produção por área deste experimento, conduzido em pastagem tropical.

Os resultados obtidos para P ainda são superiores aos observados por Koger \& Euclides Filho (1985) em relação às vacas Shorthorn (média de $87 \mathrm{~kg}$ de 
bezerros desmamados/vaca) e semelhante ao desempenho de vacas Brahman (média de $129 \mathrm{~kg}$ ). No entanto, são inferiores aos das vacas mestiças (P de $142 \mathrm{~kg}$ de bezerro), cujas médias foram resultantes também do uso de pastagens nativa, parcialmente melhorada e pastagem melhorada.

\section{Conclusões}

Elevadas taxas de produtividade, de eficiência e de eficiência por hectare foram obtidas com vacas da raça Nelore em pastagem de Brachiaria decumbens Stapf adubada, com livre acesso à mistura mineral, nas condições ambientais dos Cerrados do Brasil Central.

Altas taxas de repetição de prenhez ocorrem em vacas com média de peso de $405 \mathrm{~kg}$ e 3,3 de condição corporal durante a estação de acasalamento.

A ordem de parto das vacas tem efeito sobre o peso à desmama. Vacas primíparas e velhas desmamam bezerros mais leves.

\section{Literatura Citada}

ABREU, J.C. Sobre o peso ao nascer na raça Nelore. Revista da Agricultura, v.24, n.7/8, p.231-251, 1949.

ALBUQUERQUE, L.G.; ELER, J.P.; COSTA, M.J.R.P. et al. Produção de leite e desempenho do bezerro na fase de aleitamento em três raças bovinas de corte 1. Revista Brasileira de Zootecnia, v.22, n.5, p.745-754, 1993.

AROEIRA, J.A.D.C.; ROSA, A.N. Desempenho reprodutivo de um rebanho Nelore criado no planalto SulMatogrossense. Pesquisa Agropecuária Brasileira, v.17, n.2, p.337-343, 1982.

BARBOSA, C.; CAMPOS, B.E.S.; CÉSAR, S.M. et al. Distribuição do nascimento de bovinos da raça Nelore submetidos a monta natural o ano todo, na região de Araçatuba (SP). Boletim da Indústria Animal, v.35, n.1, p.1-15, 1978.

BARCELLOS, J.O.J.; LOBATO, J.F.P.; FRIES, L.A. Eficiência de vacas primíparas Hereford e cruzas Hereford - Nelore acasaladas no outono/inverno ou na primavera/verão. Revista Brasileira de Zootecnia, v.25, n.3, p.414-427, 1996.

BERETTA, V.; LOBATO, J.F.P.; MIELITZ NETTO, C.G.A. Produtividade e eficiência biológica de sistemas pecuários de cria diferindo na idade das novilhas ao primeiro parto e na taxa de natalidade do rebanho no Rio Grande do Sul. Revista Brasileira de Zootecnia, v.30, n.4, p.1278-1286, 2001.

BERETTA, V.; LOBATO, J.F.P.; MIELITZ NETTO, C.G.A. Produtividade e eficiência biológica de sistemas de produção de gado de corte de ciclo completo no Rio Grande do Sul. Revista Brasileira de Zootecnia, v.31, n.2, p.991-1001, 2002 (Supl.).

CARDELliNO, R.A.; CASTRO, L.F.S. Efeitos ambientais e fatores para peso ao nascer, peso a desmama e ganho de peso pré-desmama em bovinos Nelore. Revista Brasileira de Zootecnia, v.16, n.1, p.14-27, 1987.
CEZAR, I. M. Modelo bioecônomico de produção de bovinos de corte. I. Descrição do modelo. Pesquisa Agropecuária Brasileira, v.17, n.6, p.941-949, 1982.

CEZAR, I.M. Sistemas de produção de novilho precoce. In: ENCONTRO NACIONAL SOBRE NOVILHO PRECOCE, Campinas, 1995. Anais... Campinas: CATI, 1995. p.39-55. CORREAA, E.S.; ANDRADE P.; EUCLIDES FILHO, K. et al. Avaliação de um sistema de gado de corte. 1. Desempenho reprodutivo. Revista Brasileira de Zootecnia, v.29, n.6, p.220- 2215, 2000 (Supl. 2).

CORRÊA, E.S.; EUCLIDES FILHO, K.; ALVES, R.G.O. et al. Desempenho reprodutivo em um sistema de produção de gado de corte. Campo Grande: EMBRAPA/CNPGC, 2001. 33p. (Boletim de Pesquisa, 13)

DODE, M.A.N.; VALLE, E.R.; ROSA, G.O. Efeito da interrupção temporária do aleitamento sobre a fertilidade de vacas de corte. Revista Brasileira de Reprodução Animal, v.13, n.2, p.109-120, 1989.

ELER, J.P.; LÔBO, R.B; ROSA, A.N. Influência de fatores genéticos e de meio em pesos de bovinos da raça Nelore criados no Estado de São Paulo. Revista Brasileira de Zootecnia, v.18, n.2, p.103-111, 1989.

EMPRESA BRASILEIRA DE PESQUISA AGROPECUÁRIA EMBRAPA. Serviço Nacional de Levantamento e Conservação de Solos. Levantamento de reconhecimento detalhado e aptidão agrícola dos solos da área do Centro Nacional de Pesquisa de Gado de Corte, Mato Grosso do Sul. Rio de Janeiro, 1979. 225p. (Boletim Técnico, 59)

EUClides FILHO, K. Produção de bovinos de corte e o trinômio - ambiente - mercado. Campo Grande: EMBRAPA/CNPGC, 2000. 61p. (Documentos, 85)

EUCLIDES FILHO, K.; FIGUEIREDO, G.R.; EUCLIDES, V.P.B. Eficiência de produção de vacas de corte com diferentes potenciais para produção de leite. Pesquisa Agropecuária Brasileira, v.30, n.7, p.1003-1007, 1995.

EUCLIDES FILHO, K.; FIGUEIREDO, G.R.; SILVA, L.O.C. et al. Idade aos $165 \mathrm{~kg}$ de peso vivo para progênies de Nelore, Fleckvieh, Chianina, Charolês, f1's e retrocruzas. Revista Brasileira de Zootecnia, v.27, n.5, p.899-905, 1998.

FAGUNDES, J.I.B.; LOBATO, J.F.P.; SCHENKEL, F.S. Efeito de duas cargas animais em campo nativo e de duas idades à desmama no desempenho de vacas de corte primíparas. Revista Brasileira de Zootecnia, v.32, n.6, p.1722-1731, 2003 (supl. 1).

FERREIRA, P.R.C.; SAMPAIO, I.B.M. Fatores responsáveis por variações de peso de bezerros Nelore. Arquivos da Escola de Veterinária da UFMG, v.33, n.3, p.515-518, 1981.

KOGER, M.; EUCLIDES FILHO, K. Genetics aspects of the relationship between cow type and response to nutritional environment. In: SIMPÓSIO INTERNACIONAL DE PRODUÇÃO ANIMAL, 1985, Ribeirão Preto. Anais... Ribeirão Preto: Sociedade Brasileira de Genética, 1985. p.1-10.

LEME, P.R.; BOIN, C.; NARDON, R.F. Efeito da estação de monta e da taxa de lotação no desempenho reprodutivo de vacas de corte. Boletim da Indústria Animal, v.46, n.1, p.133-141, 1989.

LIMA, F.P. Estudo de alguns fatores de variação do peso à desmama dos bezerros de raças zebuínas de corte. Boletim da Indústria Animal, v.31, n.1, p.39-65, 1974.

LOBATO, J.F.P. Tecnologias necessárias para a pecuária de corte eficiente e competitiva. In: CICLO DE PALESTRAS EM PRODUÇÃO E MANEJO DE BOVINOS, 6., Canoas, 2001. Anais... Canoas: ULBRA, 2001. v.1, p.29-48. 
LOWMAN, B.G.; SCOTT, N.A.; SOMERVILLE, S.H. Condition scoring of cattle. Edimburg: East of Scotland College of Agriculture. Animal production. Advisory and Development Department, 1976. 31p. (Bulletin, 6).

MADRUGA, C.R.; GOMES, R.; SCHENK, M.A.M. et al. Etiologia de algumas doenças de bezerros de corte no Estado do Mato Grosso do Sul. Campo Grande: EMBRAPA/ CNPGC, 1984. 27p. (Circular Técnica, 15)

McMANUS, C.; SAUERESSING, M.G.; FALCÃO, R.A. et al. Componentes reprodutivos e produtivos no rebanho de corte da Embrapa Cerrados. Revista Brasileira de Zootecnia, v.31, n.2, p.648-657, 2002.

MORAES, A.A.S.; LOBATO, J.F.P. Efeito de duas épocas de desmama no desempenho reprodutivo de vacas de corte. Revista Brasileira de Zootecnia, v.22, n.6, p.1003$1011,1993$.

PACOLA, L.J.; BOIN, C.; RAZOOK, A.G. Suplementação protéica, no período da seca, para vacas zebuínas primíparas (1). Boletim da Indústria Animal, v.40, n.2, p.201-206, 1983.

PIMENTEL, D.M.; ZIMMER, A.H. Capim setária: características e aspectos produtivos. [S.1.]: EMBRAPA/CNPGC, 1983. 71p. (Documentos, 11)

PÖTTER, B.A.A.; LOBATO, J.F.P. Efeitos de carga animal, pastagem melhorada e da idade de desmame no comportamento reprodutivo de vacas primíparas. Revista Brasileira de Zootecnia, v.33, n.1, p.192-202, 2004.

RUAS, J.R.M.; TORRES, C.A.A.; VALADARES FILHO, S.C. et al. Efeito da suplementação protéica a pasto sobre o consumo de forragens, ganho de peso e condição corporal, em vacas Nelore. Revista Brasileira de Zootecnia, v.29, n.3, p.930-934, 2000.

SANTIAGO, M.; NASCIMENTO, J.; ALENCAR, M.M. et al. Bovinos da raça Canchim submetidos a dois períodos de monta: estudo comparativo das taxas de fertilidade e desmama. Boletim da Indústria Animal, v.40, n.2, p.189-193, 1983.

STATISTICAL ANALYSES SYSTEM - SAS. SAS/STAT user's guide. 4.ed. Version 6, Cary: 1990. v.2, 1686p.
SAUERESSIG, M.G.; ROCHA, C.M.C. Manejo da reprodução de gado de corte na região dos Cerrados. Planaltina: EMBRAPA/CPAC, 1985.CPAC.6p. (Comunicado Técnico, 44)

SILVA, L.O.C.; ROSA, A.N.; NOBRE, P.R.C. et al. Análise de pesos de bovinos Nelore criados a pasto no Estado de São Paulo, Brasil. Pesquisa Agropecuária Brasileira, v.22, n.11/12, p.1245-1256, 1987.

SIMEONE, A.; LOBATO, J.F.P. Efeito da lotação animal em campo nativo e do controle da amamentação no comportamento reprodutivo de vacas de corte primíparas. Revista Brasileira de Zootecnia, v.25, n.6, p.1216-1227, 1996.

S.THIAGO, L.R.L.; MORAES, S.S.; NICODEMO, M.L.F. et al. Efeito do fósforo suplementar sobre o desempenho reprodutivo de vacas de corte em pastagem de Brachiaria humidicola . Pesquisa Agropecuária Brasileira, v.35, n.2, p.449-456, 2000.

TREWARTHA, G.T. An introdution to climate. 3.ed. New York: McGraw-Hill, 1954. p.381-383.

WILTBANK, J.N.; ROWDEN, W.W.; INGALLS, J.E. et al. Effect of energy level on reproductive phenomena of mature Hereford cows. Journal of Animal Science, v.21, n.3, p.219-225, 1962.

WILTBANK, J.N. Body condition scoring in beef catlle. In: NAYLON, J.; RALSTON, S.L. (Eds.) Large animal clinical nutrition. St. Louis: Mosby, 1991. p.164-178.

ZIMMER, A.H.; EUCLIDES FILHO, K. As Pastagens e a Pecuária de Corte Brasileira. In: SIMPÓSIO INTERNACIONAL SOBRE PRODUÇÃO ANIMAL EM PASTEJO,1997, Viçosa, MG. Anais... Viçosa, MG: [s.n.], 1997. p.349-380.

ZIMMER, A.H.; EUCLIDES, V.P.B.; EUCLIDES FILHO, K. et al. Considerações sobre índices de produtividade da pecuária de corte em Mato Grosso do Sul. Campo Grande: EMBRAPA/CNPGC, 1998. 53p. (Documentos, 70)

Recebido em: 09/02/04

Aceito em: 06/04/05 